\title{
OPTIMIZATION OF DIFFERENTIAL SYSTEMS WITH HYSTERESIS
}

\author{
Jürgen Sprekels \\ Weierstrass Institute for Applied Analysis and Stochastics, \\ Mohrenstrasse 39, D - 10117 Berlin, Germany \\ sprekels@wias-berlin.de
}

Dan Tiba

Weierstrass Institute for Applied Analysis and Stochastics, Mohrenstrasse 39, D - 10117 Berlin, Germany, and Institute of Mathematics, Romanian Academy, P. O. Box 1-764, RO-70700 Bucharest, Romania tiba@wias-berlin.de and dtiba@imar.ro

Abstract We investigate general control problems governed by ordinary differential systems involving hysteresis operators. Our main hypotheses are of continuity type, and we discuss existence results, discretization methods, and approximation approaches.

Keywords: Peano-type existence theorem, continuous hysteresis operators, Clarke's generalized gradient

\section{Introduction}

Many engineering systems contain nonlinear functional dependencies of hysteresis type. We quote the recent monographs by Visintin [16] and by Brokate and Sprekels [6] for a mathematical treatment of the topic. Concerning the control of such systems basic references are the book of Brokate [2] and his articles [3], [4], [5], and the works of Smith [14], Banks, Smith and Wang [1].

We analyze a controlled ordinary differential system with hysteresis:

$$
\begin{gathered}
z^{\prime}=f(t, z, y, u) \quad \text { in }[0, T] \\
z(0)=z_{0} \in \mathbb{R}^{N} \\
y(t)=W(S[z])(t), \quad S[z](t)=g(z(t)) \text { in }[0, T],
\end{gathered}
$$

The original version of this chapter was revised: The copyright line was incorrect. This has been corrected. The Erratum to this chapter is available at DOI: 10.1007/978-0-387-35690-7_44

V. Barbu et al. (eds.), Analysis and Optimization of Differential Systems

(c) IFIP International Federation for Information Processing 2003 


$$
u(t) \in U \quad \text { in }[0, T] .
$$

Here $f:[0, T] \times \mathbb{R}^{N} \times \mathbb{R} \times \mathbb{R}^{m} \rightarrow \mathbb{R}^{N}$ and $g: \mathbb{R}^{N} \rightarrow \mathbb{R}$ are continuous mappings, $U \subset \mathbb{R}^{m}$ is a closed bounded convex set, and $W: C[0, T] \rightarrow C[0, T]$ is a hysteresis operator, i.e. rate-independent and with the Volterra property, Sprekels and Brokate [6].

To the relations (1.1)-(1.4) the following cost functional is associated

$$
J(u)=\int_{0}^{T} L(y(t), z(t), u(t)) d t
$$

with $L: \mathbb{R} \times \mathbb{R}^{N} \times U \rightarrow \mathbb{R}$ continuous in $y, z$, convex and lower semicontinuous with respect to $u$.

We allow $g$ or $S$ to be nonlinear, and many of the results that we shall establish will use just continuity and not require local Lipschitz assumptions on the data. Our investigation has the main motivation to provide a theoretically founded way towards the approximation and the numerical analysis of the control problem (1.1)-(1.5). In this respect, it seems that only the paper by Brokate [3] reports numerical experiments in a control problem with hysteresis. While in that work the optimality conditions are solved numerically, our approach uses a complete discretization of (1.1)-(1.5) and the computation of descent directions via the Clarke [8] generalized gradient. This allows the application of bundle-type algorithms, Strodiot and Nguyen [15], Lemaréchal [11].

In Section 2 we give the formulation of the control problem, and we establish the existence of optimal controls under general assumptions. Section 3 introduces the fully discretized optimization problem and studies existence and approximation questions. It is shown that the mapping control $\mapsto$ state is Lipschitz under the given assumptions on $f, g, W$.

Section 4 uses an alternative formulation of the problem to analyze the variations and the directional derivatives. An algorithm and examples are also indicated.

\section{Existence}

We start with a Peano-type result for the Cauchy problem with hysteresis (1.1)-(1.3). We first omit the dependence on $u$, and we assume that $f:[0, T] \times \mathbb{R}^{N} \times \mathbb{R} \rightarrow \mathbb{R}^{N}, g: \mathbb{R}^{N} \rightarrow \mathbb{R}$, and $W: C[0, T] \rightarrow$ $C[0, T]$ are continuous mappings and operators. Notice that $W$ remains causal and rate-independent, i.e.:

$$
\begin{gathered}
\left.v_{1}\right|_{[0, t]}=\left.v_{2}\right|_{[0, t]} \Rightarrow W\left(v_{1}\right)(t)=W\left(v_{2}\right)(t), \quad t \in[0, T], \\
W(v \circ \varphi)(t)=W(v)(\varphi(t)), \quad \forall v \in C[0, T],
\end{gathered}
$$


for any admissible time transformation $\varphi:[0, T] \rightarrow[0, T]$, continuous, nondecreasing, and onto.

Theorem 2.1 Under the above assumptions, the initial value problem (1.1) - (1.3) has at least one global solution $z \in C^{1}\left([0, T] ; \mathbb{R}^{N}\right)$ if the following sublinearity hypotheses are fulfilled:

$$
\begin{gathered}
|W(w)|_{C[0, T]} \leq \alpha+\beta|w|_{C[0, T]}, \quad \forall w \in C[0, T] \\
|f(t, z, y)|_{\mathbb{R}^{N}} \leq \alpha+\beta|z|_{\mathbb{R}^{N}}+\gamma|y| \\
|g(y)| \leq \alpha+\gamma|y|, \quad \alpha, \beta, \gamma \in \mathbb{R}_{+}
\end{gathered}
$$

Proof. For the positive numbers $a, b$ and $c=\alpha+\beta q, q=\max \{|g(z)|$; $\left.\left|z-z_{0}\right|_{\mathbb{R}^{N}} \leq b\right\}$, consider the set

$$
\Delta=\left\{0 \leq t \leq a,\left|z-z_{0}\right|_{\mathbb{R}^{N}} \leq b,|y| \leq c\right\} .
$$

Denote by $M=\max \left\{|f(t, z, y)|_{\mathbb{R}^{N}} ;(t, z, y) \in \Delta\right\}$ and $\delta=\inf \left(a, \frac{b}{M}, \frac{c}{M}\right)$. Clearly $f$ is uniformly continuous in $\Delta$, that is, $|f(t, z, y)-f(\tilde{t}, \tilde{z}, \tilde{y})|_{\mathbb{R}^{N}}<\varepsilon$ for any $\varepsilon>0$ if $(t, z, y),(\tilde{t}, \tilde{z}, \tilde{y}) \in \Delta$ and $|t-\tilde{t}|<\eta(\varepsilon),|z-\tilde{z}|_{\mathbb{R}^{N}}<\eta(\varepsilon)$, $|y-\tilde{y}|<\eta(\varepsilon)$. Denote by $h_{\varepsilon}=\inf \left(\eta(\varepsilon), \frac{\eta(\varepsilon)}{M}\right)$, and take the division $t_{j}=j h_{\varepsilon}, j \in \mathbb{N}$, of $[0, \delta]$.

We consider the polygonal functions (the Picard iterations with Euler polygonal lines):

$$
\begin{aligned}
\varphi_{\varepsilon}(t) & =\varphi_{\varepsilon}\left(t_{j}\right)+\left(t-t_{j}\right) f\left(t_{j}, \varphi_{\varepsilon}\left(t_{j}\right), y_{\varepsilon}\left(t_{j}\right)\right), t_{j}<t \leq t_{j+1}, \\
\varphi_{\varepsilon}(0) & =z_{0} \\
y_{\varepsilon}\left(t_{j}\right) & =W_{f}\left(S\left(\varphi_{\varepsilon}\right)\right)\left(t_{j}\right) .
\end{aligned}
$$

Due to (2.1), relation (2.5) makes sense. Recall that

$$
W_{f}\left(S\left(\varphi_{\varepsilon}\right)\right)\left(t_{j}\right)=\tilde{W}_{f}\left(S\left(z_{0}\right), S\left(\varphi_{\varepsilon}\left(t_{1}\right)\right), \ldots, S\left(\varphi_{\varepsilon}\left(t_{j}\right)\right)\right)
$$

with $W_{f}: C[0, T] \rightarrow \mathbb{R}$ being the generating functional of $W$, Brokate and Sprekels [6]. Here, $\mathcal{S}$ is the set of all finite strings of real numbers and $\tilde{W}_{f}$ the application induced on $\mathcal{S}$ by $W_{f}$ (see Section 3 ).

Note that (2.2) plays an essential role in this construction. Then:

$$
\begin{aligned}
\left|\varphi_{\varepsilon}\left(t_{j+1}\right)-z_{0}\right|_{\mathbb{R}^{N}} & \leq\left|\varphi_{\varepsilon}\left(t_{j}\right)-z_{0}\right|_{\mathbb{R}^{N}}+h_{\varepsilon}\left|f\left(t_{j}, \varphi_{\varepsilon}\left(t_{j}\right), y_{\varepsilon}\left(t_{j}\right)\right)\right| \\
& \leq\left|\varphi_{\varepsilon}\left(t_{j}\right)-z_{0}\right|_{\mathbb{R}^{N}}+M h_{\varepsilon} .
\end{aligned}
$$

Here, we argue by induction:

Assuming that $\left(t_{j}, \varphi_{\varepsilon}\left(t_{j}\right), y_{\varepsilon}\left(t_{j}\right)\right) \in \Delta$ and $\left|\varphi_{\varepsilon}\left(t_{j}\right)-z_{0}\right|_{\mathbb{R}^{N}} \leq j M h_{\varepsilon}$, relation (2.7) gives that $\left|\varphi_{\varepsilon}\left(t_{j+1}\right)-z_{0}\right| \leq(j+1) M h_{\varepsilon} \leq M \delta \leq b$. 
By (2.3), (2.7), and (2.7), we have

$$
\left|y_{\varepsilon}\left(t_{j}\right)\right| \leq \alpha+\beta \max _{0 \leq i \leq j}\left|S\left(\varphi_{\varepsilon}\left(t_{i}\right)\right)\right|_{\mathbb{R}^{N}} \leq \alpha+\beta q=c .
$$

Inequalities (2.7), (2.9) show that $\left(t_{j}, \varphi_{\varepsilon}\left(t_{j}\right), y_{\varepsilon}\left(t_{j}\right)\right) \in \Delta$ in all steps (2.5) such that $j h_{\varepsilon} \in[0, \delta]$. Then, $\left|f\left(t_{j}, \varphi_{\varepsilon}\left(t_{j}\right), y_{\varepsilon}\left(t_{j}\right)\right)\right|_{\mathbb{R}^{N}} \leq M$, and (2.5) gives directly that

$$
\left|\varphi_{\varepsilon}(t)-\varphi_{\varepsilon}(s)\right|_{\mathbb{R}^{N}} \leq M|t-s| \leq \eta(\varepsilon), \forall \varepsilon>0, \forall t, s \in[0, \delta] .
$$

Then $\varphi_{\varepsilon} \rightarrow \varphi$ uniformly in $[0, \delta]$, on a subsequence, and $\varphi \in$ $C\left([0, \delta] ; \mathbb{R}^{N}\right)$. If $\tilde{y}_{\varepsilon}(t)=W\left(S\left(\varphi_{\varepsilon}\right)\right)(t)$, then $\tilde{y}_{\varepsilon}\left(t_{j}\right)=y_{\varepsilon}\left(t_{j}\right)$ and $\tilde{y}_{\varepsilon} \rightarrow$ $y=W(S(\varphi))$ in $C[0, \delta]$ due to the continuity of $S, W$, and on the same subsequence. We also have that

$$
\left.\varphi_{\varepsilon}^{\prime}(t)=f\left(t_{j}, \varphi_{\varepsilon}\left(t_{j}\right), y_{\varepsilon}\left(t_{j}\right)\right) \text { if } t \in\right] t_{j}, t_{j+1}[,
$$

while, by $(2.10)$,

$$
\left|f\left(t_{j}, \varphi_{\varepsilon}\left(t_{j}\right), y_{\varepsilon}\left(t_{j}\right)\right)-f\left(t, \varphi_{\varepsilon}(t), y_{\varepsilon}(t)\right)\right|_{\mathbb{R}^{N}} \leq \varepsilon, t \in\left[t_{j}, t_{j+1}\right]
$$

by the uniform continuity of $f$ in $\Delta$.

For any $\lambda>0$, again the uniform continuity of $f$ in $\Delta$ gives that

$$
\left|f\left(t, \varphi_{\varepsilon}(t), y_{\varepsilon}\left(t_{j}\right)\right)-f\left(t, \varphi_{\varepsilon}(t), \tilde{y}_{\varepsilon}(t)\right)\right|_{\mathbb{R}^{N}} \leq \lambda, \quad t \in\left[t_{j}, t_{j+1}\right],
$$

if $\varepsilon<\varepsilon(\lambda)$, due to the equicontinuity of the sequence $\left\{\tilde{y}_{\varepsilon}\right\}$ and to $\tilde{y}_{\varepsilon}\left(t_{j}\right)=y_{\varepsilon}\left(t_{j}\right)$.

Relations (2.11) $-(2.13)$ allow to pass to the limit and to see that $\varphi, y$ give a solution of $(1.1)-(1.3)$ in $[0, \delta]$ and that $\varphi \in C^{1}[0, \delta]$.

Under assumptions (2.3)-(2.5), it is well-known that the local solution is in fact a global one, Brokate and Sprekels [6], p. 126.

Remark. By Theorem 2.1, uniqueness may be not true for the state system (1.1)-(1.3). The control problem (1.1)-(1.5) has to be understood as a minimization over pairs: to each control we associate all the possible states. This is well-known in the setting of optimal control theory of ODEs, Cesari [7], or for singular control problems for PDEs, Lions [12].

We assume that the continuity of $L(\cdot, \cdot, u): \mathbb{R} \times \mathbb{R}^{N} \rightarrow \mathbb{R}_{+}$is uniform with respect to $u \in U$. The mapping $f$ is affine with respect to $u$, i.e.

$$
f(t, z, y, u)=f_{1}(t, z, y)+f_{2}(t, z, y) u
$$

where $f_{1}:[0, T] \times \mathbb{R}^{N} \times \mathbb{R} \rightarrow \mathbb{R}^{N}, f_{2}:[0, T] \times \mathbb{R}^{N} \times \mathbb{R} \rightarrow \mathbb{R}^{m \times N}$ are continuous sublinear mappings (like in (2.4)). 
As $U$ is bounded, convex and closed, the admissible controls are in $L^{\infty}\left([0, T] ; \mathbb{R}^{m}\right)$, and the mapping (2.14) will not satisfy the continuity requirements of Theorem 2.1 (with respect to $t$, via $u$ ). However, the argument from its proof can be repeated when $u$ is continuous, and a simple approximation argument may be used for $u \in L^{\infty}\left([0, T] ; \mathbb{R}^{m}\right)$. The state $z \in W^{1, \infty}\left([0, T] ; \mathbb{R}^{N}\right)$ in this case.

Theorem 2.2 Under the above assumptions, the optimal control problem (1.1) - (1.5) has at least one optimal triplet

$$
\left[u^{*}, z^{*}, y^{*}\right] \in L^{\infty}([0, T] ; U) \times W^{1, \infty}\left([0, T] ; \mathbb{R}^{N}\right) \times C[0, T] .
$$

Proof. Let $\left[u_{n}, z_{n}, y_{n}\right]$ be a minimizing sequence. Then $\left\{u_{n}\right\}$ is bounded in $L^{\infty}\left([0, T] ; \mathbb{R}^{m}\right)$. By $(2.4),(2.14)$ and $(1.1),(1.2)$, we have

$$
\begin{aligned}
\left|z_{n}(\hat{t})\right|_{\mathbb{R}^{N}} & \leq\left|z_{0}\right|_{\mathbb{R}^{N}}+\int_{0}^{\hat{t}}\left|f\left(t, z_{n}(t), y_{n}(t), u_{n}(t)\right)\right|_{\mathbb{R}^{N}} d t \\
& \leq\left|z_{0}\right|_{\mathbb{R}^{N}}+c \int_{0}^{t}\left(\alpha+\beta\left|z_{n}(t)\right|_{\mathbb{R}^{N}}+\gamma\left|y_{n}(t)\right|\right) d t .
\end{aligned}
$$

We may consider the truncated functions $\tilde{y}_{n}, \tilde{z}_{n}$ to the interval $[0, t]$, and we still have $\tilde{y}_{n}(t)=y_{n}(t)=W\left(S\left(\tilde{z}_{n}\right)\right)(t)$. Then (2.3) applies, and we get:

$$
\begin{aligned}
\left|z_{n}(\hat{t})\right|_{\mathbb{R}^{N}} & \leq\left|z_{0}\right|_{\mathbb{R}^{N}}+c \int_{0}^{\hat{t}}\left(\alpha+\beta\left|z_{n}(t)\right|_{\mathbb{R}^{N}}+\alpha+\beta \sup _{s \in[0, t]}\left|z_{n}(s)\right|_{\mathbb{R}^{N}}\right) d t \\
& \leq\left|z_{0}\right|_{\mathbb{R}^{N}}+2 c \alpha \hat{t}+2 c \beta \int_{0}^{\hat{t}} \sup _{s \in[0, t]}\left|z_{n}(s)\right|_{\mathbb{R}^{N}} d t .
\end{aligned}
$$

Taking the supremum in both sides of the inequality above, we obtain

$$
\sup _{t \in[0, \hat{t}]}\left|z_{n}(t)\right|_{\mathbb{R}^{N}} \leq\left|z_{0}\right|_{\mathbb{R}^{N}}+2 c \alpha T+2 c \beta \int_{0}^{\hat{t}} \sup _{s \in[0, t]}\left|z_{n}(s)\right|_{\mathbb{R}^{N}} d t
$$

and Gronwall's lemma shows that $\left\{z_{n}\right\}$ is bounded in $C\left([0, T] ; \mathbb{R}^{N}\right)$. By (1.1), (1.2), (2.14) we see that $\left\{z_{n}\right\}$ is bounded in $W^{1, \infty}\left([0, T] ; \mathbb{R}^{N}\right)$. And (2.3), (2.5), (1.3) give that $\left\{y_{n}\right\}$ is bounded in $C[0, T]$.

We denote $u_{n} \rightarrow \bar{u} \in U$ weakly* in $L^{\infty}\left([0, T] ; \mathbb{R}^{m}\right)$ and $z_{n} \rightarrow \bar{z}$ in $C\left([0, T] ; \mathbb{R}^{N}\right)$, on a subsequence. Then, the continuity of $W, S$ gives that $y_{n} \rightarrow \bar{y}$ in $C[0, T]$ with $\bar{y}=W(S(\bar{z}))$.

Moreover, (2.4) and (2.14), via the Lebesgue theorem, ensure that

$$
\int_{0}^{\hat{t}} f\left(t, z_{n}(t), y_{n}(t), u_{n}(t)\right) d t \rightarrow \int_{0}^{\hat{t}} f(t, \bar{z}(t), \bar{y}(t), \bar{u}(t)) d t
$$


which shows that $[\bar{u}, \bar{z}, \bar{y}]$ satisfies (1.1)-(1.4), i.e. it is admissible for the control problem. Notice that

$$
\left|\int_{0}^{T} L\left(z_{n}(t), y_{n}(t), u_{n}(t)\right) d t-\int_{0}^{T} L\left(\bar{z}(t), \bar{y}(t), u_{n}(t)\right) d t\right| \rightarrow 0
$$

as the continuity of $L\left(\cdot, \cdot, u_{n}\right)$ is uniform with respect to $u_{n}$. Thus:

$$
\liminf _{n \rightarrow \infty} \int_{0}^{T} L\left(\bar{z}(t), \bar{y}(t), u_{n}(t)\right) d t \geq \int_{0}^{T} L(\bar{z}(t), \bar{y}(t), \bar{u}(t)) d t
$$

by the convexity and lower semicontinuity with respect to $u$ of $L$ and the weak* convergence of $u_{n}$.

The two last convergence properties show that $[\bar{u}, \bar{z}, \bar{y}]$ is optimal for the problem (1.1)-(1.5), and the proof is finished.

\section{Discretization}

Consider an equidistant partition $\left\{t_{i}\right\}_{i=\overline{0, k}}$ of $[0, T]$ of step-size $\Delta t>0$. The discretized control problem is

$$
\operatorname{Min}\left\{\sum_{j=1}^{k+1} L\left(y_{j}, z_{j}, u_{j}\right) \Delta t\right\}
$$

subject to $u_{i+1} \in U, i=\overline{0, k}$ and

$$
\begin{aligned}
& z_{i+1}=z_{i}+\Delta t f\left(t_{i+1}, z_{i+1}, y_{i+1}, u_{i+1}\right), \\
& y_{i+1}=\tilde{W}_{f}\left(S\left(z_{0}\right), S\left(z_{1}\right), \ldots, S\left(z_{i+1}\right)\right) .
\end{aligned}
$$

Here, $\tilde{W}_{f}(s)=W_{f}\left(\pi_{A}(s)\right), \forall s \in \mathcal{S}$, the set of all finite strings of real numbers, and $z_{0}$ is given. The application $\pi_{A}(s)$ is the piecewise linear interpolation operator with equidistant nodes in $[0, T]$ corresponding to the number of components of $s \in \mathcal{S}$, and $W_{f}$ is the generating functional associated to $W$, Brokate and Sprekels [6]. The definition of $\tilde{W}_{f}$ is essentially based on (2.2). We have the following result.

Proposition 3.1 If $f, g, \tilde{W}_{f}$ are continuous in their arguments and satisfy (2.3)-(2.5), then for every $\left\{u_{i+1}\right\}_{\overline{0, k}} \in U^{k+1}$, the equations (3.1), (3.2) have at least one solution $\left\{z_{i+1}\right\}_{\overline{0, k}} \in\left[\mathbb{R}^{N}\right]^{k+1},\left\{y_{i+1}\right\}_{\overline{0, k}} \in \mathbb{R}^{k+1}$.

Proof. The argument is iterative, for every $i$. Assuming the solution defined at level $i$ is known, then $z_{i+1} \in \mathbb{R}^{N}$ and $y_{i+1} \in \mathbb{R}$ are obtained as a fixed point of the continuous application (in finite dimensional spaces) defined by the right-hand side of (3.1). 
If $B_{i+1}$ is a "big" closed ball around 0 , in $\mathbb{R}^{N}$, containing $z_{0}, z_{1}, \ldots, z_{i}$ in the interior of $\frac{1}{2} B_{i+1}$, then relations (2.3)-(2.5) show that $z_{i}+\Delta t f\left(t_{i+1}, z, y, u_{i+1}\right) \in B_{i+1}$ for $\Delta t$ "small" if $z \in B_{i+1}$ and $y=\tilde{W}_{f}\left(S\left(z_{0}\right), S\left(z_{1}\right), \ldots, S\left(z_{i}\right), S(z)\right)$. Then, Brouwer's fixed point theorem, Kelley [10], provides at least one solution $z_{i+1}$ of (3.1), and $y_{i+1}=\tilde{W}_{f}\left(S\left(z_{0}\right), S\left(z_{1}\right), \ldots, S\left(z_{i}\right), S\left(z_{i+1}\right)\right)$.

The important remark in this argument is that, for $z \in B_{i+1}$, then $|z|_{\mathbb{R}^{N}} \leq r_{i+1}$ (the radius), and (2.3)-(2.5) generate a constant $\Gamma>0$, independent of $i$, such that $\left|f\left(t_{i+1}, z, y, u_{i+1}\right)\right|_{\mathbb{R}^{N}} \leq \Gamma r_{i+1}$. Then $\Delta t=\frac{T}{k}<(2 \Gamma)^{-1}$ will be a satisfactory choice of $\Delta t$ in the above argument, which is also independent of $i$.

Corollary 3.2 Under the above assumptions, if $L$ is convex and lower semicontinuous in $u$ and continuous in $y, z$ uniformly with respect to $u$, then the discrete control problem $\left(\mathbf{P}_{\mathrm{k}}\right)$ has at least one optimal n-tuple $\left[\left(u_{j}^{k}\right),\left(z_{j}^{k}\right),\left(y_{j}^{k}\right)\right]_{j=\overline{1, k+1}}$ in $U^{k+1} \times\left(\mathbb{R}^{N}\right)^{k+1} \times \mathbb{R}^{k+1}$.

The argument is similar to that used in the proof of Theorem 2.2, and we omit it.

Obviously $\left\{u_{i}^{k}\right\}$ are bounded in $\mathbb{R}^{m}$ for any $k$ and for $i=\overline{1, k+1}$. We examine the boundedness properties of $\left\{z_{i}^{k}\right\},\left\{y_{i}^{k}\right\}$.

By (2.4), (3.1), and the boundedness of $U$, we get

$$
\left|z_{i+1}^{k}\right|_{\mathbb{R}^{N}} \leq\left|z_{i}^{k}\right|_{\mathbb{R}^{N}}+C \Delta t\left(1+\left|z_{i+1}^{k}\right|_{\mathbb{R}^{N}}+\left|y_{i+1}^{k}\right|\right) .
$$

We also have, by (3.2), (2.3), (2.5), that

$$
\left|y_{i+1}^{k}\right| \leq C\left(1+\max _{0 \leq j \leq i+1}\left|z_{j}^{k}\right|_{\mathbb{R}^{N}}\right) \text {. }
$$

By (3.3), (3.4), taking the maximum with respect to the indices, we see

$$
\max _{0 \leq j \leq i+1}\left|z_{j}^{k}\right|_{\mathbb{R}^{N}} \leq \max _{0 \leq j \leq i}\left|z_{j}^{k}\right|_{\mathbb{R}^{N}}+C \Delta t\left(1+2 \max _{0 \leq j \leq i+1}\left|z_{j}^{k}\right|_{\mathbb{R}^{N}}\right) .
$$

Here $C$ is an "absolute" constant depending just on $\alpha, \beta, \gamma$ from (2.3)(2.5) and on the bound of $\left\{u_{i}^{k}\right\}$ in $\mathbb{R}^{m}$.

Summing (2.5) with respect to $i$, we can infer that

$$
\max _{0 \leq j \leq i+1}\left|z_{j}^{k}\right|_{\mathbb{R}^{N}} \leq \sum_{l=0}^{i} C \Delta t\left(1+2 \max _{0 \leq j \leq l+1}\left|z_{j}^{k}\right|_{\mathbb{R}^{N}}\right) .
$$

If $\Delta t$ is "small", the discrete Gronwall inequality shows that $\left\{z_{i}^{k}\right\}$ are bounded in $\mathbb{R}^{N}$ with respect to $k$ and to $i=\overline{0, k+1}$. Inequality (3.4) gives the same for $\left\{y_{i}^{k}\right\}$ in $\mathbb{R}$. 
Let us now construct the $\varphi_{k}$ as in the proof of Theorem 2.1:

$$
\left.\left.\varphi_{k}^{\prime}(t)=f\left(t_{i+1}, \varphi_{k}\left(t_{i+1}\right), y_{k}\left(t_{i+1}\right), u_{k}\left(t_{i+1}\right)\right), \quad t \in\right] t_{i+1}, t_{i+2}\right] .
$$

The mapping $y_{k}(t)=W\left(S\left(\varphi_{k}\right)\right)(t)$ and clearly $\left\{\varphi_{k}\right\}$ is bounded in $W^{1, \infty}\left([0, T] ; \mathbb{R}^{N}\right),\left\{y_{k}\right\}$ is bounded in $C[0, T]$, and $u_{k}$ (piecewise constant interpolation of $\left.u_{i}^{k}\right)$ is bounded in $L^{\infty}\left([0, T] ; \mathbb{R}^{m}\right)$.

We thus have $\varphi_{k} \rightarrow \hat{\varphi}$ uniformly in $C\left([0, T] ; \mathbb{R}^{N}\right)$, on a subsequence. By the continuity of $S$ and of $W$, we get $y_{k} \rightarrow \hat{y}=W(S \hat{\varphi})$ in $C[0, T]$. We also may assume $u_{k} \rightarrow \hat{u}$, on the same subsequence, weakly* in $L^{\infty}\left([0, T] ; \mathbb{R}^{m}\right)$.Now, compute the difference

$$
\begin{aligned}
& D=f\left(t, \varphi_{k}(t), u_{k}(t)\right)-f\left(t_{i+1}, \varphi_{k}\left(t_{i+1}\right), y_{k}\left(t_{i+1}\right), u_{k}\left(t_{i+1}\right)\right), \\
& \left.t \in] t_{i+1}, t_{i+2}\right]
\end{aligned}
$$

and take into account that $u_{k}(t) \equiv u_{k}\left(t_{i+1}\right)$ in this interval, and (2.14). The uniform continuity of $f_{1}, f_{2}$ and the above uniform convergences show that $|D| \leq \varepsilon$ if $k$ is big enough. One can pass to the limit to see that $\hat{\varphi}, \hat{y}, \hat{u}$ is an admissible pair for the original control problem, i.e. it satisfies (1.1)-(1.4). By comparing with the cost obtained in $\left(\mathbf{P}_{\mathbf{k}}\right)$ via the discretization of $u^{*}$ (provided by Theorem 2.2) and by passing to the limit, we also have:

Theorem 3.3 The triplet $[\hat{u}, \hat{\varphi}, \hat{y}]$ is optimal for the problem (1.1) (1.5).

The next two statements concern Lipschitz properties of the mappings that we are using. We omit the proofs which are rather direct.

Lemma 3.4 If $W: C[0, T] \rightarrow C[0, T]$ is Lipschitz of rank $C>0$ and $s_{1}=\left(v_{0}, v_{1}, \ldots, v_{l}\right), s_{2}=\left(w_{0}, w_{1}, \ldots, w_{l}\right) \in \mathcal{S}$ have the same number of components, then

$$
\left|\tilde{W}_{f}\left(v_{0}, v_{1}, \ldots, v_{l}\right)-\tilde{W}_{f}\left(w_{0}, w_{1}, \ldots, w_{l}\right)\right| \leq C \max _{0 \leq j \leq l}\left|v_{j}-w_{j}\right| .
$$

Remark. Under regularity/Lipschitz assumptions on $f, g, L$, Lemma 3.4 shows that the functional dependence from $\left\{u_{i}\right\} \in U^{k+1}$ to the cost is a Lipschitzian dependence. Thus, the Clarke [8] generalized gradient may be used to write the optimality conditions for $\left(\mathbf{P}_{\mathbf{k}}\right)$ and to devise descent algorithms. More about this will be said below.

Proposition 3.5 Assume that, $f, g$ are real Lipschitz mappings and that $W$ is a Lipschitz operator in $C[0, T]$. Then the correspondence $\left\{u_{i}\right\} \mapsto\left\{z_{i}\right\}$ defined by $(3.1),(3.2)$ is Lipschitz from $\left(\mathbb{R}^{m}\right)^{k+1}$ to $\left(\mathbb{R}^{N}\right)^{k+1}$. 
Remark. If the differentiable mapping $\tilde{S}:\left(\mathbb{R}^{N}\right)^{i+1} \rightarrow \mathbb{R}^{i+1}$, $\tilde{S}\left(z_{0}, z_{1}, \ldots, z_{i+1}\right)=\left(S\left(z_{0}\right), S\left(z_{1}\right), \ldots, S\left(z_{i+1}\right)\right)$ has a surjective Jacobian, then the chain rule is valid for the Clarke generalized gradient " $\partial$ " of the composed mapping $\tilde{W}_{f}(\tilde{S})$, Clarke et al. [9], Theorem 3.2:

$$
\partial\left(\tilde{W}_{f} \circ \tilde{S}\right)(\cdot)=\left[\tilde{S}^{\prime}(\cdot)\right]^{*} \partial \tilde{W}_{f}(\tilde{S}(\cdot)),
$$

and one can write the first-order optimality conditions for $\left(\mathbf{P}_{\mathbf{k}}\right)$.

\section{Approximation}

In this section, in order to fix ideas, we shall assume that the mapping $L$ is quadratic and independent of $y$. We shall perform a further approximation of the problem $\left(\mathbf{P}_{\mathbf{k}}\right)$ by the penalization of (3.2) into the cost. We do not regularize the hysteresis operator $W$, as in Brokate [2]. Roughly speaking, we shall interpret $y$ as a supplementary/artificial control, and (3.2) as a mixed control-state constraint.

Since in the theory of hysteresis operators, Brokate and Sprekels [6], the piecewise monotonicity of mappings plays an important role, our penalization method uses just the positive part function, $(\cdot)_{+}$, which is monotone:

$$
\begin{aligned}
\operatorname{Min} & \left\{\frac{1}{2} \sum_{j=1}^{k+1}\left|z_{j}-z_{d}\left(t_{j}\right)\right|_{\mathbb{R}^{N}}^{2}\left(t_{j+1}-t_{j}\right)+\frac{1}{2} \sum_{j=1}^{k+1}\left|u_{j}\right|_{\mathbb{R}^{m}}^{2}\left(t_{j+1}-t_{j}\right)\right. \\
+ & \frac{1}{\varepsilon} \max _{j=\overline{1, k+1}}\left[\left(y_{j}-\tilde{W}_{f}\left(S\left(z_{0}\right), \ldots, S\left(z_{j}\right)\right)\right)_{+} ;\right. \\
& \left.\left.\left(\tilde{W}_{f}\left(S\left(z_{0}\right), \ldots, S\left(z_{j}\right)\right)-y_{j}\right)_{+}\right]\right\}
\end{aligned}
$$

subject to $u_{j} \in U, y_{j} \in \mathbb{R},(3.1)$, and with $z_{0}$ given as an initial condition.

The approximation properties of the penalized problem (4.1) with respect to $\left(\mathbf{P}_{\mathbf{k}}\right)$, when $\varepsilon \rightarrow 0$, are standard, and we do not discuss this here.

Moreover, under usual differentiability and Lipschitz assumptions on $f$ in (3.1) the mapping $\left\{y_{i}\right\} \in \mathbb{R}^{k+1},\left\{u_{i}\right\} \in\left(\mathbb{R}^{m}\right)^{k+1} \mapsto\left\{z_{i}\right\} \in\left(\mathbb{R}^{N}\right)^{k+1}$ is differentiable and Lipschitz for the corresponding finite dimensional norms.

Consequently, we may view (4.1) as the minimization of a Lipschitzian real mapping ( $\tilde{W}_{f}$ is just Lipschitz), depending on $\left\{u_{i}\right\}$ and $\left\{y_{i}\right\}$, and under the constraint $u_{i} \in U$, convex, closed, bounded subset in $\mathbb{R}^{m}$. 
We have already seen that the Clarke generalized gradient of $\tilde{W}_{f}(\tilde{S}(\cdot))$ may be computed via the chain rule (3.9). This may be done directly with respect to $\left\{u_{i}\right\}$ and $\left\{y_{i}\right\}$, since the dependence (3.1) of $\left\{z_{i}\right\}$ on these variables, denoted as $\mathcal{A}: \mathbb{R}^{k+1} \times\left(\mathbb{R}^{m}\right)^{k+1} \rightarrow\left(\mathbb{R}^{N}\right)^{k+1}$, may be assumed $C^{1}$, and having a surjective Jacobian.

Let us denote shortly by $\mathcal{C}=\tilde{W}_{f} \circ \tilde{S} \circ \mathcal{A}: \mathbb{R}^{k+1} \times\left(\mathbb{R}^{m}\right)^{k} \rightarrow \mathbb{R}$, the superposition Lipschitzian mapping. It is to be noticed that the composition of $\mathcal{C}$ with $(\cdot)_{+}$, appearing in (4.1), does not fulfil the assumptions of the chain rules indicated in Clarke et al. [9], Ch. 2.4. In particular, the mapping $\mathcal{C}$ is not regular, in general (i.e. the generalized Clarke directional derivative may not coincide with the usual directional derivative). However, the mapping $(\cdot)_{+}$is regular (since it is convex), has positive gradients and, clearly, a very simple structure. A direct computation may be used to establish the following result.

Proposition 4.1 If $y_{i}-\mathcal{C}\left(\left\{u_{i}\right\},\left\{y_{i}\right\}\right)>0$, and if $\left[\left\{v_{i}\right\},\left\{x_{i}\right\}\right]$ is some variation of $\left[\left\{u_{i}\right\},\left\{y_{i}\right\}\right]$, respectively, then

$$
\begin{aligned}
& \limsup _{\left.\left\{\left\{\tilde{u}_{i}\right\},\left\{\tilde{y}_{i}\right\}\right\} \rightarrow\left\{u_{i}\right\},\left\{y_{i}\right\}\right\}} \frac{\left(\tilde{y}_{i}+\lambda x_{i}-\mathcal{C}\left(\left[\left\{\tilde{u}_{i}\right\},\left\{\tilde{y}_{i}\right\}\right]+\lambda\left[\left\{v_{i}\right\},\left\{x_{i}\right\}\right]\right)\right)_{+}-\left(\tilde{y}_{i}-\mathcal{C}\left(\left\{\tilde{u}_{i}\right\},\left\{\tilde{y}_{i}\right\}\right)\right)_{+}}{\lambda} \\
& =\limsup _{\left.\left\{\left\{\tilde{u}_{i}\right\},\left\{\tilde{y}_{i}\right\}\right\} \rightarrow\left\{u_{i}\right\},\left\{y_{i}\right\}\right]} \frac{\tilde{y}_{i}+\lambda x_{i}-\mathcal{C}\left(\left[\left\{\tilde{u}_{i}\right\},\left\{\tilde{y}_{i}\right\}\right]+\lambda\left[\left\{v_{i}\right\},\left\{x_{i}\right\}\right]\right)-\tilde{y}_{i}+\mathcal{C}\left(\left\{\tilde{u}_{i}\right\},\left\{\tilde{y}_{i}\right\}\right)}{\lambda} . \\
& \text { If } y_{i}-\mathcal{C}\left(\left\{u_{i}\right\},\left\{y_{i}\right\}\right)<0, \text { the above limsup is null. }
\end{aligned}
$$

Remark. By Clarke et al. [9], p. 79, if $\tilde{S}$ and $\mathcal{A}$ are $C^{1}$ with surjective Jacobians, then the last limsup in Proposition 4.1 coincides with

$$
\limsup _{\substack{w \rightarrow \tilde{S}\left(\mathcal{A}\left(\left\{u_{i}\right\},\left\{y_{i}\right\}\right)\right) \\ \lambda \downarrow 0}}\left[-\frac{\tilde{W}_{f}\left(w+\lambda(\tilde{S} \circ \mathcal{A})^{\prime}\left(\left\{v_{i}\right\},\left\{x_{i}\right\}\right)\right)-\tilde{W}_{f}(w)}{\lambda}\right]+x_{i} .
$$

Here, $(\tilde{S} \circ \mathcal{A})^{\prime}$ denotes the Jacobi matrix of the composed mapping. Relation (4.2) and Proposition 4.1 indicate how to compute the Clarke generalized directional derivative of the cost functional (4.1). For the max-operation appearing in (4.1), one has to take the maximum of the limsup computed as above.

The main example that we consider concerns the case when $W$ is the so-called play operator. We introduce the real mapping (for some given $r>0$ )

$$
f_{r}(v, w)=\max \{v-r, \min \{v+r, w\}\}, \quad \forall v, w \in \mathbb{R} .
$$

Taking into account the discretized problems (3.2) or (4.1), we define directly the mapping $\tilde{W}_{f}$ on $\mathcal{S}$. This can be done inductively, Brokate 
and Sprekels [6], p. 39:

$$
\begin{gathered}
\tilde{W}_{f}\left(v_{0}\right)=f_{r}\left(v_{0}, w\right), \quad \forall v_{0} \in \mathbb{R}, \\
\tilde{W}_{f}\left(v_{0}, \ldots, v_{i}\right)=f_{r}\left(v_{i}, \tilde{W}_{f}\left(v_{0}, \ldots, v_{i-1}\right)\right), \quad \forall v_{0}, \ldots, v_{i} \in \mathbb{R} .
\end{gathered}
$$

Here $w \in \mathbb{R}$ is fixed (one can take $w=0$ ) and has the significance of an initial (or memory) condition imposed on the operator $\tilde{W}_{f}$.

Proposition 4.2 The nonlinear functional $\tilde{W}_{f}$ is piecewise linear on $\mathbb{R}^{i}$, for any given $i$.

This is an immediate consequence of (4.3)-(4.5). We underline that piecewise linear functionals are neither regular in the sense of Clarke [8], nor weakly semismooth in the sense of Mifflin [13].

However, Proposition 4.2 and (3.9) show that it is possible to compute numerically the Clarke generalized gradient $\partial \tilde{W}_{f}(\tilde{S} \circ \mathcal{A}(\cdot))$, in any point. The observation is that $\partial \tilde{W}_{f}(\cdot)$ is piecewise constant in $\mathbb{R}^{i}$, for any $i$ and, consequently, a finite number of operations will suffice.

It is known that $d(\cdot)=\operatorname{proj}_{\partial \tilde{W}_{f}(\tilde{S} \circ \mathcal{A}(\cdot))}\{0\}$ is a descent direction if it is nonzero, Clarke [8]. This may be tested by the computations indicated in Proposition 4.1. If it is zero, then a stationary point has been achieved.The following conceptual algorithm may be used:

\section{Algorithm 4.3}

1 Let $\left\{u_{i}\right\},\left\{y_{i}\right\}$ be given.

2 Compute the generating vectors of $\partial J\left(\left\{u_{i}\right\},\left\{y_{i}\right\}\right)$.

3 Compute $d$.

4 If $d=0$, then STOP.

5 If $d \neq 0$, then

$$
\left[\left\{u_{i}\right\},\left\{y_{i}\right\}\right] \rightarrow\left[\left\{u_{i}\right\},\left\{y_{i}\right\}\right]-\rho d, \quad \rho>0 .
$$

6 Compute $J\left(\left[\left\{u_{i}\right\},\left\{y_{i}\right\}\right]-\rho d\right)$.

7 GO TO 2.

Here $J$ is the cost defined by (4.1). We note that in Step 5, a line search has to be performed. In general, there is no convergence ensured for Algorithm 4.3 as the weak semismoothness of $J$ is not valid, Strodiot and Nguyen [15]. Therefore, in practice, a number of steps has to be prescribed or some numerical convergence tests have to be introduced. 


\section{References}

[1] H.T. Banks, R.C. Smith and Y. Wang. Smart Material Structures: Modeling, Estimation and Control. Masson, Paris, 1996.

[2] M. Brokate. Optimale Steuerung von gewöhnlichen Differentialgleichungen mit Nichtlinearitäten vom Hysteresis-Typ. Peter-Lang-Verlag, Frankfurt am Main, 1987.

[3] M. Brokate. Numerical solution of an optimal control problem with hysteresis. In LN Control and Information Sciences 95, pages 68-78, Berlin, 1987. Springer.

[4] M. Brokate. Optimal control of ODE systems with hysteresis nonlinearities. In ISNM 84, pages 25-41, Basel, 1988. Birkhäuser.

[5] M. Brokate. Optimal control of the semilinear wave equation with hysteresis. In Free Boundary Problems: Theory and Applications (K.H. Hoffmann and J. Sprekels, eds.), pages 451-458, Harlow, 1990. Longman.

[6] M. Brokate and J. Sprekels. Hysteresis and Phase Transitions. Springer, New York, 1996.

[7] L. Cesari. Optimization - Theory and Applications. Springer, Berlin, 1983.

[8] F.H. Clarke. Optimization and Nonsmooth Analysis. Wiley Interscience, New York, 1983.

[9] F.H. Clarke, Yu.S. Ledyaev, R.J. Stern and P.R. Wolonski. Nonsmooth Analysis and Control Theory. Springer Graduate Texts in Mathematics 178, New York, 1998.

[10] J.L. Kelley. General Topology. Springer, New York-Berlin, 1975.

[11] C. Lemaréchal. Bundle methods in nondifferentiable optimization. In Nonsmooth Optimization (C. Lemaréchal and R. Mifflin, eds.), pages 79-102, Oxford, 1978. Pergamon Press.

[12] J.L. Lions. Contrôle des systèmes distribués singuliers. Dunod, Paris, 1983.

[13] R. Mifflin. An algorithm for constrained optimization with semi-smooth functions. Math. Oper. Res., 2:191-207, 1977.

[14] R.C. Smith. Hysteresis modeling in magnetostrictive materials via Preisach operators. J. Math. Systems Estim. Control, 8(2):23 pp. (electronic), 1998.

[15] J.J. Strodiot and V.H. Nguyen. On the numerical treatment of the inclusion $0 \in \partial f(x)$. In Topics in Nonsmooth Mechanics (J.J. Moreau, P.D. Panagiotopoulos, G. Strang, eds.), pages 267-294, Basel, 1988. Birkhäuser.

[16] A. Visintin. Differential Models of Hysteresis. Springer, Berlin, 1994. 